\title{
Potential antiviral effects of some native Iranian medicinal plants extracts and fractions against influenza $A$ virus
}

\author{
Parvaneh Mehrbod ${ }^{1}$, Hanieh Safari' ${ }^{2}$ Zeinab Mollai ${ }^{2}$, Fatemeh Fotouhi ${ }^{1}$, Yasaman Mirfakhraei ${ }^{2}$, Hanieh Entezari²,
} Saied Goodarzi ${ }^{3^{*}}$ and Zahra Tofighi ${ }^{2,3^{*}}$ (1)

\begin{abstract}
Background: Influenza A virus (IAV) infection is a continual threat to the health of animals and humans globally. Consumption of the conventional drugs has shown several side effects and drug resistance. This study was aimed to screen some Iranian medicinal plants extracts and their fractions against influenza A virus.

Methods: Glycyrrhiza glabra (rhizome), Myrtus commonis (leaves), Melissa officinalis (leaves), Hypericum perforatum (aerial parts), Tilia platyphyllos (flower), Salix alba (bark), and Camellia sinensis (green and fermented leaves) were extracted with $80 \%$ methanol and fractionated with chloroform and methanol, respectively. The cytotoxicity of the compounds were determined by MTT colorimetric assay on MDCK cells. The effective concentrations $\left(E_{50}\right)$ of the compounds were calculated from the MTT results compared to the negative control with no significant effects on cell viability. The effects of $\mathrm{EC}_{50}$ of the compounds on viral surface glycoproteins and viral titer were tested by $\mathrm{HI}$ and $\mathrm{HA}$ virological assays, respectively and compared with oseltamivir and amantadine. Preliminary phytochemical analysis were done for promising anti-IAV extracts and fractions.

Results: The most effective samples against IAV titer $(P \leq 0.05)$ were crude extracts of $G$. glabra, $M$. officinalis and $S$. alba; methanol fractions of $M$. communis and M. officinalis; and chloroform fractions of $M$. communis and $C$. sinensis (fermented) mostly in co- and pre-penetration combined treatments. The potential extracts and fractions were rich in flavonoids, tannins, steroids and triterpenoids.

Conclusion: The outcomes confirmed a scientific basis for anti-influenza A virus capacity of the extracts and fractions from the selected plants for the first time, and correlated their effects with their phytochemical constituents. It is worth focusing on elucidating pure compounds and identifying their mechanism(s) of action.
\end{abstract}

Keywords: Licorice, Lemon balm, White willow, Myrtle, Black tea, Anti-influenza A viral activity

*Correspondence: Goodarzi_S@tums.ac.ir; ztofighi@tums.ac.ir

2 Department of Pharmacognosy, Faculty of Pharmacy, Tehran University of Medical Sciences, Tehran, Iran

${ }^{3}$ Medicinal Plants Research Center, Faculty of Pharmacy, Tehran University of Medical Sciences, Tehran, Iran

Full list of author information is available at the end of the article

\section{Background}

Influenza A virus (IAV) is one of the most severe respiratory diseases which leads to the high rates of morbidity and mortality $[1,2]$. Vaccination, as a peventive method, can not provide sufficient control against the spread of this infection because of continuous antigenic drifts [3]. The adamantane derivatives including amantadine and rimantadine were used for the treatment and prophylaxis of influenza disease for several years [4]. Oseltamivir 
$\left(\right.$ Tamiflu $\left.^{\circledR}\right)$, as FDA-approved drug, which is a neuraminidase inhibitor molecule with the basic structure of shikimic acid was isolated and purified from the fruits of Illicium verum Hook. f. (Illiciaceae) [5]. Treatment with conventional drugs including amantadine and oseltamivir has shown side effects on the central nervous system and the gastrointestinal tract. Also, because of the genetic instability and reassortments of the virus from one side and drug resistance from the other side, the prescription of these drugs has been inefficient in some cases [6-8]. Due to the need for having other inhibitors of IAV, the scientists have focused on the screening medicinal plants and natural products and to find and introduce a lead compound or structure for the further preclinical trials [9].

Medicinal plants play vital roles in prevention and treatment of several diseases. The natural products and medicinal plants are popular in different parts of the world from developing to developed countries as complementary to common medicines [10,11]. Medicinal plants are cheaper, more available and culturally acceptable especially in Iran with a strong and long history of traditional medicine in comparison with some chemical drugs with undesirable adverse effects [12].

There are several plants used from ancient times till now for the treatment of common cold, flu syndrom and infections in throat and upper respiratory systems. Although there were many ethnopharmacology articles about them, a few reports highlighted their anti-influenza virus effects $[13,14]$.

To the best of our knowledge, there are no reports about antiviral effects of G. glabra, M. commonis, S. alba, T. platyphyllos and C. sinensis (fermented) extracts against influenza A virus. Also, there are no reports about anti-influenza effects of fractions of other mentioned plants. The objective of this study was to investigate the potential effects of crude extracts and different fractions of some Iranian medicinal plants against influenza replication, and compare their activities with oseltamivir and amantadine as control drugs. For this purpose, the propagation and cytopathic effects of IAV in the presence of different extracts and fractions were determined using the hemagglutination (HA), hemagglutination inhibition (HI), and MTT cytotoxicity assays. The potent extract(s) and fraction(s) can be introduced as sources of phytochemicals with antiviral effects against influenza A virus.

\section{Methods}

\section{Preparation of plant extracts and fractions}

Rhizomes of Glycyrrhiza glabra (Leguminosae, PMP246), leaves of Myrtus commonis (Myrtaceae, PMP-421) and Melissa officinalis (Labiatae, PMP-358), aerial parts of Hypericum perforatum (Hypericaceae, PMP-526), and bark of Salix alba (Salicaceae, PMP-924) were purchased in July 2017 from market in Tehran, Iran. Dried and fermented leaves of Camellia sinensis (Theaceae, PMP-415 and PMP-416, respectively) were obtained in November 2017 from market of Lahijan, Iran. Flowers of Tilia platyphyllos (Malvaceae, 7057-TEH) were collected from Ramsar, Mazandaran, Iran. The voucher speciments were identified by Dr. Zahra Tofighi and Dr. Yousef Ajani, and deposited in herbarium of Faculty of Pharmacy, Tehran University of Medical Sciences, Tehran, Iran. All dried plants were extracted with $80 \%$ methanol and fractionated with chloroform to give chloroform fractions and the residues were named methanol fractions.

\section{Cell culture and influenza virus propagation}

Madin Darby Canine Kidney (MDCK) cells were maintained in Dulbecco's Modified Eagle's Medium (DMEM) (Mediatech Cellgro, USA) containing 10\% Fetal Bovine Serum (FBS) (PAA, Austria) and 1\% Penicillin and streptomycin (Mediatech Cellgro, USA) in $5 \% \mathrm{CO}_{2}$ incubator at $37^{\circ} \mathrm{C}$. Influenza virus strain [A/Puerto Rico/8/1934 (H1N1) (ATCC VR-1469 $9^{\mathrm{TM}}$ )] was prepared from Influenza Department, Pasteur Institute of Iran, and propagated in MDCK cells. DMEM supplemented with $1 \mu \mathrm{g} /$ $\mathrm{ml}$ of Trypsin-TPCK (Tosylamide Phenylethyl Chloromethyl Keton-treated Trypsin) (Sigma, USA) was used as maintenance medium during antiviral experiments. For measuring the virus infectivity dose, the $50 \%$ cell culture infectious dose $\left(\mathrm{CCID}_{50}\right)$ was obtained by the hemagglutination assay using Karber method [15, 16].

\section{Cytotoxicity assay}

Two-fold serial dilutions of the extracts and fractions were exposed to the sub-cultured MDCK cells in 96-well plates $\left(3 \times 10^{4}\right.$ cell/well), for $48 \mathrm{~h}$ at $37^{\circ} \mathrm{C}$ in duplicates. The test was repeated two times. The colorimetric MTT assay was performed as previously described by Mehrbod et al. [16]. Briefly, the culture medium was replaced by MTT 1X [3-(4,5-dimethyl-2-thiazolyl)-2,5-diphenyl$2 \mathrm{H}$-tetrazolium bromide; Sigma, USA]. Following 3-4h incubation at $37^{\circ} \mathrm{C}$ in dark, DMSO $(100 \mu \mathrm{l})$ was added to each well to release the purple color of formazan. The absorbance at $570 \mathrm{~nm}$ was measured with a microplate reader (BioTek EL 800, US). The cell viability was calculated based on the following formula: (mean Optical Density (OD) of treated cells/mean OD of control cells $) \times 100$. The $50 \%$ cytotoxic concentration $\left(\mathrm{CC}_{50}\right)$, the concentration that reduced the cell viability by $50 \%$ respect to the control cells; and the $50 \%$ effective concentration $\left(\mathrm{EC}_{50}\right)$, the concentration required to achieve $50 \%$ protection against virus induced cytopathic effect, were also calculated by analyzing MTT data using SPSS software. The cells without any exposure which were 
considered as negative controls and DMSO as a vehicle control with maximum $0.5 \%$ concentration were tested in parallel. Amantadine hydrochloride and oseltamivir carboxylate, the conventional antiviral compounds, were also tested as positive control drugs.

\section{Selectivity index}

The selectivity index (SI) calculated by dividing $\mathrm{CC}_{50}$ to $\mathrm{EC}_{50}$, represents the relative safety of the extracts or frections. It has been demonstrated that compounds with selectivity indices higher than 3 are potentially safe antiviral reagents [17].

\section{Antiviral assay}

In co-penetration procedure of antiviral evaluations, influenza virus $\left(100 \mathrm{TCID}_{50} / 0.1 \mathrm{ml}\right)$ was mixed with the $\mathrm{EC}_{50}$ of the extracts and fractions for $30 \mathrm{~min}$, and then incubated with the cells at $37^{\circ} \mathrm{C}$. In pre-penetration and post-penetration procedures, the virus was added to the cells after and before the extracts and fractions. Following $1 \mathrm{~h}$ incubation, the unabsorbed viruses were replaced by TPCK-containing medium $(1 \mu \mathrm{g} / \mathrm{ml})$. Following $48 \mathrm{~h}$ incubation at $37^{\circ} \mathrm{C}$, viabilities of the cells were measured by MTT assay as described earlier. Concurrently, the virus titer was determined by testing the cell supernatants using the HA assay [16]. Amantadine hydrochloride $(98.5 \mu \mathrm{g} / \mathrm{ml})$ and oseltamivir carboxylate $(394.25 \mu \mathrm{g} / \mathrm{ml})$ (Sigma, Saint Louis, Missouri, USA) were considered as positive control drugs and the cells without any exposure were tested as negative controls. DMSO was tested as a vehicle control with maximum $0.5 \%$ concentration. The test was repeated two times in duplicates.

\section{Cellular percentage of protection}

The protection percentages of the samples were calculated by SPSS from the MTT data results of mockinfected and infected cells after $48 \mathrm{~h}$ exposure, using the following formula: Percentage of protection $=[(\mathrm{ODT})$ $\mathrm{V}-(\mathrm{ODC}) \mathrm{V}] /[(\mathrm{ODC}) \mathrm{M}-(\mathrm{ODC}) \mathrm{V}] \times 100$ where $(\mathrm{ODT}) \mathrm{V},(\mathrm{ODC}) \mathrm{V}$ and $(\mathrm{ODC}) \mathrm{M}$ represent the absorbance of the treated sample, the virus-infected control (no compound) and the negative control (mock), respectively [18].

\section{Hemagglutination assay (HA)}

For quantification of the virus titer from the cell supernatants, the HA assay was conducted as previously described [19]. Briefly, serial dilutions of the culture media were added to 96-well U-shaped microplates in duplicates. The test was repeated two times. The HA units were measured as the reciprocal of the highest dilution giving complete agglutination with chicken red blood cells $(0.5 \%)$. The precipitation and diffuse lattice formation of the RBCs demonstrate the absence and the presence of the virus, respectively.

\section{Hemagglutination inhibition assay (HI)}

For investigation of the inhibitory effect of the samples on the hemagglutinin activity, the concentration of 4 HA unit of virus particles was used. Briefly, the extracts and fractions were diluted 2-fold serially from $\mathrm{CC}_{50}$ concentration. Then, $4 \mathrm{HA}$ unit of the virus was added to each well. After pre-incubation for $45 \mathrm{~min}$ at room temperature, chicken red blood cells $(0.5 \%)$ were added to the solution. The physical interaction between extracts/fractions and virus surface HA glycoprotein was calculated after $1 \mathrm{~h}$ by the agglutination inhibition pattern.

\section{Preliminary phytochemical analysis}

Crude extracts, chloroform and methanol fractions of the effective anti-influenza plants were tested for identifying the class of active metabolites such as alkaloids, cardiac glycosides, tannins, flavonoids, steroids, triterpenoids and saponins by following the standard procedures $[20$, 21].

\section{Tests for alkaloids}

A) Dragendorff's test: The apperance of reddish brown turbidity after addition of Dragendorff reagent was indicator for the presence of alkaloids.

B) Hager's test: By addition of Hager reagent to the samples, yellow turbidity was seen in the presence of alkaloids.

\section{Tests for cardiac glycosides}

Keller-killiani test: The mixture of ferric chloride and glacial acetic acid were added to the sample solutions. Apperance of bluish green in the upper layer and reddish color in the lower layer of the solutions, after addition of concentrated sulphuric acid confirmed the existence of cardiac glycosides in the samples.

\section{Test for tannins}

$\mathrm{FeCl}_{3}$ test: After adding a few drops of $5 \% \mathrm{w} / \mathrm{v}$ solution of ferric chloride III in $90 \%$ alcohol to the sample solutions, the appearance of deep blue or dark green color indicated the tannin existence in the samples.

\section{Test for flavonoids (Shinoda test)}

After adding few drops of concentrated $\mathrm{HCl}$ and magnesium metal to the samples, the appearance of pink, red, crimson or magenta color was the sign of flavonoids presentation. 


\section{Tests for steroids and triterpenoids}

Libermann-Buchard test: The appearance of brown ring in the middle of green upper layer and deep red lower layer after addition of sulfuric acid containing few drops of acetic anhydride were indicator for the presence of steroids and triterpenoids.

Salkowski test: By addition of chloroform and concentrated $\mathrm{H}_{2} \mathrm{SO}_{4}$ to the extract and shaking well, the color of chloroform layer changed to red, and acid layer showed greenish yellow fluorescent if sterols and/or triterpenes existed.

\section{Test for saponins (foam test)}

The volume of $50 \mathrm{mg}$ of samples in water was shaken vigorously in a test tube. If froth characteristic was obtained, the presence of saponins was confirmed.

\section{Statistical analysis}

The data were expressed as mean $\pm \mathrm{SD}$, and analyzed by one-way analysis of variance (ANOVA) and General Linear Model (GLM) (SPSS 18.0), Tukey and Duncan post-hoc tests. Sample values with $P \leq 0.05$ and $P \leq 0.01$ were considered statistically significant and highly significant, respectively.

\section{Results}

In this study, the efficacy of the crude extracts, chloroform and methanol fractions of 8 selected plants with history of usages in traditinal and folklore Iranian medicine were tested against IAV. The cytotoxicity of the extracts were evaluated, and non-toxic concentrations were defined prior to antiviral assay. The ability of the samples against viral titer and viral cytopathic effects varied with different extracts and fractions of the same plant as determined by the HA and MTT assays. The profile of the extracts and fractions of selected medicinal plants used in this study is listed in Table 1.

\section{Cytotoxicity and selectivity indices of the compounds}

The results for $\mathrm{CC}_{50}$ were largely different for various samples (Table 2). Among the crude extracts, the highest $\mathrm{CC}_{50}$ value belonged to $S$. alba $(3647.45 \pm 52.52 \mu \mathrm{g} /$ $\mathrm{ml})$ and the lowest $\mathrm{CC}_{50}$ value $(10.41 \pm 0.00 \mu \mathrm{g} / \mathrm{ml})$ was obtained for $M$. communis and $H$. perforatum equally. Among the chloroform fractions, $S$. alba and $M$. communis showed the highest $(1755.28 \pm 0.89 \mu \mathrm{g} /$ $\mathrm{ml})$ and the lowest $(2.60 \pm 0.00 \mu \mathrm{g} / \mathrm{ml}) \mathrm{CC}_{50}$ values, respectively. Amongst methanol fractions the highest and the lowest $C_{50}$ values were obtained for $M$. officinalis $(4413.80 \pm 3.69 \mu \mathrm{g} / \mathrm{ml})$ and T. platyphyllos $(277.56 \pm 3.19 \mu \mathrm{g} / \mathrm{ml})$, respectively. The $\mathrm{EC}_{50}$ values which were the same as non-cytotoxic concentrations
Table 1 Profile of the medicinal plants extraction

\begin{tabular}{llll}
\hline Botanical Name & $\begin{array}{l}\text { Extract } \\
\text { yeilds } \\
\text { (\%) }\end{array}$ & Chl F. Yeild (\%) & Met F. Yeild (\%) \\
\hline Glycyrrhiza glabra & 27.2 & 16.4 & 75.9 \\
Myrtus communis & 39.4 & 19.8 & 72.9 \\
Melissa officinalis & 42.2 & 16.5 & 79.8 \\
Hypericum perforatum & 39.1 & 28.0 & 66.2 \\
Tilia platyphyllos & 11.9 & 41.5 & 55.3 \\
Salix alba & 15.5 & 6.6 & 57.1 \\
Camellia sinensis & 36.8 & 55.4 & 41.4 \\
Camellia sinensis (fer- & 25.0 & 40.0 & 60.0 \\
mented) & & & \\
\hline
\end{tabular}

Chl F. Chloroform Fraction, Met F. Methanol Fraction

(NCTC) with no significant effects on the cell viability were calculated using data obtained from MTT and one-way ANOVA analysis as compared to the negative control (Table 2). The selectivity index values were obtained with the highest SI value of 32 with $M$. officinalis (chloroform fraction), $H$. perforatum (methanol fraction), $S$. alba (crude extract and methanol fraction) and C. sinensis unfermented (methanol fraction) and the lowest value of 2 with G. glabra (chloroform fraction).

\section{Inhibitory effect of extracts and fractions on influenza a virus}

The samples were tested in an in vitro screening assay to define the antiviral activity against IAV. The antiviral activity of the plants extracts/fractions was analyzed based on the $\log _{10}$ HA titer (Table 3) and Log HA decrement (Table 4). Among them, in co-penetration treatments, G. glabra (crude extract; $5.00 \pm 4.24$ ), G. glabra (methanol fraction; 5.50土3.54), M. communis (chloroform fraction; $5.50 \pm 3.54$ ) and $M$. officinalis (methanol fraction; $6.00 \pm 2.83)$; in pre-penetration treatment, $M$. communis (methanol fraction; $5.50 \pm 0.71$ ); and in postpenetration treatment: $M$. communis (chloroform fraction; $5.50 \pm 3.54)$ showed highly significant $(P \leq 0.01)$ decrease in HA titer compared to the virus inoculation. These results were in accordance with the Log HA decrement (Table 5) which showed 5-6 logs decrement in Log HA titer for all samples.

\section{Cell viability and cellular percentage of protection}

The optical densities (ODs) in MTT assay for the antiviral activity against IAV revealed highly significant increments in the majority of the combined treatments compared to the virus-inoculated cells $(P \leq 0.01)$. Amantadine and oseltamivir combined exposures also resulted 
Table 2 Anti-influenza A virus effects of some medicinal plants extracts and fractions

\begin{tabular}{|c|c|c|c|c|c|}
\hline Plant & Extracts or Fractions & Concentration in DMSO & $\mathrm{CC}_{50}(\mu \mathrm{g} / \mathrm{ml})$ & $\begin{array}{l}\text { NCTC }(\mu \mathrm{g} / \mathrm{ml}) \& \\
\mathrm{EC}_{50}(\mu \mathrm{g} / \mathrm{ml})\end{array}$ & $\begin{array}{l}\text { Selectivity index } \\
\left(\mathrm{SI}=\mathrm{CC}_{50} / \mathrm{EC}_{50}\right)\end{array}$ \\
\hline Glycyrrhiza glabra & Crude E. & $100 \mathrm{mg} / \mathrm{ml}$ & $881.87 \pm 0.03$ & 55.12 & 16 \\
\hline Glycyrrhiza glabra & Chloroform F. & $100 \mathrm{mg} / \mathrm{ml}$ & $5.19 \pm 0.00$ & 2.57 & 2 \\
\hline Glycyrrhiza glabra & Methanol F. & $100 \mathrm{mg} / \mathrm{ml}$ & $3529.48 \pm 0.76$ & 441.12 & 8 \\
\hline Myrtus communis & Crude E. & $100 \mathrm{mg} / \mathrm{ml}$ & $10.40 \pm 0.00$ & 2.60 & 4 \\
\hline Myrtus communis & Chloroform F. & $100 \mathrm{mg} / \mathrm{ml}$ & $2.60 \pm 0.00$ & 0.65 & 4 \\
\hline Myrtus communis & Methanol F. & $100 \mathrm{mg} / \mathrm{ml}$ & $603.67 \pm 0.60$ & 37.73 & 16 \\
\hline Melissa officinalis & Crude E. & $100 \mathrm{mg} / \mathrm{ml}$ & $1813.99 \pm 0.23$ & 113.37 & 16 \\
\hline Melissa officinalis & Chloroform F. & $100 \mathrm{mg} / \mathrm{ml}$ & $1427.10 \pm 0.45$ & 44.62 & 32 \\
\hline Melissa officinalis & Methanol F. & $100 \mathrm{mg} / \mathrm{ml}$ & $4413.80 \pm 3.69$ & 551.72 & 8 \\
\hline Hypericum perforatum & Crude E. & $100 \mathrm{mg} / \mathrm{ml}$ & $10.41 \pm 0.00$ & 1.30 & 8 \\
\hline Hypericum perforatum & Chloroform F. & $100 \mathrm{mg} / \mathrm{ml}$ & $10.99 \pm 0.00$ & 2.75 & 4 \\
\hline Hypericum perforatum & Methanol F. & $100 \mathrm{mg} / \mathrm{ml}$ & $3929.08 \pm 2.47$ & 122.7 & 32 \\
\hline Tilia platyphyllos & Crude E. & $100 \mathrm{mg} / \mathrm{ml}$ & $152.26 \pm 0.50$ & 9.56 & 15.92 \\
\hline Tilia platyphyllos & Chloroform F. & $100 \mathrm{mg} / \mathrm{ml}$ & $267.45 \pm 8.8$ & 16.69 & 16.03 \\
\hline Tilia platyphyllos & Methanol F. & $100 \mathrm{mg} / \mathrm{ml}$ & $277.56 \pm 3.19$ & 34.62 & 8.02 \\
\hline Salix alba & Crude E. & $100 \mathrm{mg} / \mathrm{ml}$ & $3647.45 \pm 52.52$ & 113.70 & 32 \\
\hline Salix alba & Chloroform F. & $100 \mathrm{mg} / \mathrm{ml}$ & $1755.28 \pm 0.89$ & 219.37 & 8 \\
\hline Salix alba & Methanol F. & $100 \mathrm{mg} / \mathrm{ml}$ & $2816.86 \pm 77.80$ & 88.03 & 32 \\
\hline Camellia sinensis & Crude E. & $100 \mathrm{mg} / \mathrm{ml}$ & $161.89 \pm 2.30$ & 20.25 & 8 \\
\hline Camellia sinensis & Chloroform F. & $100 \mathrm{mg} / \mathrm{ml}$ & $13.28 \pm 0.00$ & 1.62 & 8.17 \\
\hline Camellia sinensis & Methanol F. & $100 \mathrm{mg} / \mathrm{ml}$ & $877.28 \pm 3.80$ & 27.41 & 32 \\
\hline Camellia sinensis)fermented( & Crude E. & $100 \mathrm{mg} / \mathrm{ml}$ & $1038.192 \pm 88.73$ & 129.87 & 8 \\
\hline Camellia sinensis)fermented( & Chloroform F. & $100 \mathrm{mg} / \mathrm{ml}$ & $875.61 \pm 7.55$ & 109.50 & 8 \\
\hline Camellia sinensis)fermented( & Methanol F. & $100 \mathrm{mg} / \mathrm{ml}$ & $405.13 \pm 3.71$ & 50.62 & 8 \\
\hline Amantadine hydrochloride & - & $2000 \mu \mathrm{g} / \mathrm{ml} \mathrm{H} \mathrm{H}_{2} \mathrm{O}$ & $197.001 \pm 1.533$ & 98.500 & 2 \\
\hline Oseltamivir carboxylate & - & $4000 \mu \mathrm{g} / \mathrm{ml} \mathrm{H} \mathrm{H}_{2} \mathrm{O}$ & $788.505 \pm 6.006$ & 394.250 & 2 \\
\hline
\end{tabular}

$C C_{50} 50 \%$ cytotoxic concentration, $E C_{50} 50 \%$ effective concentration, $N C T C$ non-cytotoxic concentrations ( $E C_{50}$ and NCTC values were equal), SI Selectivity Index

in high cell viability $(P \leq 0.01)$ (Table 5$)$. The ODs in combined treatments were analyzed to obtain the percentage of compounds protection on the cell viability against the virus infectivity. Data are presented in Table 6.

In co-penetration treatments, G. glabra (methanol fraction;0.86 \pm 0.29 ), $M$. officinalis (methanol fraction; $0.81 \pm 0.05), T$. platyphyllos (methanol fraction; $0.81 \pm 0.05$ ), T. platyphyllos (chloroform fraction; $0.72 \pm 0.04$ ), S. alba (crude extract; $0.80 \pm 0.07$ ), S. alba (methanol extract; $0.66 \pm 0.07$ ), S. alba (chloroform extract; $0.87 \pm 0.11$ ), C. sinensis (chloroform extract; $0.74 \pm 0.13$ ), $C$. sinensis (fermented crude extract; $0.87 \pm 0.11$ ), C. sinensis (fermented methanol fraction; $0.80 \pm 0.04$ ), C. sinensis (fermented chloroform fraction; $0.81 \pm 0.12)$; in pre-penetration treatments, $G$. glabra (crude extract; $1.05 \pm 0.10$ ), M. officinalis (chloroform fraction; $1.01 \pm 0.31$ ), $S$. alba (crude extract; $0.73 \pm 0.03$ ), C. sinensis (chloroform fraction;
$0.81 \pm 0.16$ ), C. sinensis (fermented crude extract; $0.74 \pm 0.13$ ), C. sinensis (fermented chloroform fraction; $0.79 \pm 0.25$ ); and in post-penetration treatments, M. communis (crude extract; $0.88 \pm 0.05$ ), M. officinalis (methanol fraction; $0.84 \pm 0.09$ ), $H$. perforatum (methanol fraction; $0.79 \pm 0.06$ ), $H$. perforatum (chloroform fraction; $0.915 \pm 0.038$ ), $T$. platyphyllos (chloroform fraction; $0.68 \pm 0.07$ ), C. sinensis (methanol fraction; $0.70 \pm 0.11$ ) showed the highest cell viability and protection against virus cytopathic effects.

\section{Dose-dependent response}

The test was repeated twice. Some of the extracts and fractions showed $\mathrm{RBC}$ precipitation $(\mathrm{HI}+)$ until a certain dilution which showed dose-dependent responses for their HA physical interaction. But some other samples showed $\mathrm{RBC}$ precipitation $(\mathrm{HI}+)$ in all dilutions 
Table $3 \log _{10} \mathrm{HA}$ titer from HA assay in combined treatments with virus as compared to virus control group

\begin{tabular}{|c|c|c|c|c|}
\hline \multirow[t]{2}{*}{ Plant } & \multirow[t]{2}{*}{ Extracts or Fractions } & \multicolumn{3}{|c|}{$\log \mathrm{HA}(\operatorname{mean} \pm \mathrm{SD})$} \\
\hline & & Co-pen & Pre-pen & Post-pen \\
\hline Glycyrrhiza glabra & Crude E. & $0.90 \pm 1.28^{* *}$ & $1.20 \pm 0.43^{*}$ & $1.50 \pm 0.00$ \\
\hline Glycyrrhiza glabra & Chloroform F. & $2.11 \pm 0.00$ & $2.11 \pm 0.00$ & $1.81 \pm 0.43$ \\
\hline Glycyrrhiza glabra & Methanol F. & $0.75 \pm 1.06^{* *}$ & $1.96 \pm 0.21$ & $2.11 \pm 0.00$ \\
\hline Myrtus communis & Crude E. & $1.66 \pm 0.21$ & $1.66 \pm 0.21$ & $1.66 \pm 0.21$ \\
\hline Myrtus communis & Chloroform F. & $0.75 \pm 1.06^{* *}$ & $1.20 \pm 0.43^{*}$ & $0.75 \pm 1.06^{* *}$ \\
\hline Myrtus communis & Methanol F. & $1.50 \pm 0.43$ & $0.75 \pm 0.21^{* *}$ & $1.20 \pm 0.00^{*}$ \\
\hline Melissa officinalis & Crude E. & $1.35 \pm 0.21^{*}$ & $1.96 \pm 0.21$ & $2.11 \pm 0.00$ \\
\hline Melissa officinalis & Chloroform F. & $1.66 \pm 0.21$ & $1.66 \pm 0.21$ & $1.66 \pm 0.21$ \\
\hline Melissa officinalis & Methanol F. & $0.60 \pm 0.85^{* *}$ & $2.11 \pm 0.00$ & $1.96 \pm 0.21$ \\
\hline Hypericum perforatum & Crude E. & $1.50 \pm 0.43$ & $1.81 \pm 0.00$ & $2.11 \pm 0.00$ \\
\hline Hypericum perforatum & Chloroform F. & $1.66 \pm 0.21$ & $1.81 \pm 0.01$ & $1.66 \pm 0.21$ \\
\hline Hypericum perforatum & Methanol F. & $1.50 \pm 0.43$ & $1.66 \pm 0.21$ & $1.50 \pm 0.43$ \\
\hline Tilia platyphyllos & Crude E. & $1.81 \pm 0.00$ & $1.65 \pm 0.21$ & $1.50 \pm 0.00$ \\
\hline Tilia platyphyllos & Chloroform F. & $1.50 \pm 0.43$ & $1.66 \pm 0.21$ & $1.50 \pm 0.00$ \\
\hline Tilia platyphyllos & Methanol F. & $1.20 \pm 0.00^{*}$ & $1.96 \pm 0.21$ & $1.81 \pm 0.00$ \\
\hline Salix alba & Crude E. & $1.20 \pm 0.43^{*}$ & $1.35 \pm 0.21^{*}$ & $1.20 \pm 0.43^{*}$ \\
\hline Salix alba & Chloroform F. & $1.35 \pm 0.21^{*}$ & $1.81 \pm 0.43$ & $1.35 \pm 0.21^{*}$ \\
\hline Salix alba & Methanol F. & $1.50 \pm 0.43$ & $1.65 \pm 0.64$ & $1.65 \pm 0.64$ \\
\hline Camellia sinensis & Crude E. & $2.26 \pm 0.2$ & $1.96 \pm 0.21$ & $1.96 \pm 0.21$ \\
\hline Camellia sinensis & Chloroform F. & $1.81 \pm 0.000$ & $1.96 \pm 0.21$ & $2.11 \pm 0.43$ \\
\hline Camellia sinensis & Methanol F. & $2.26 \pm 0.21$ & $2.26 \pm 0.21$ & $1.50 \pm 0.00$ \\
\hline Camellia sinensis (fermented) & Crude E. & $1.65 \pm 0.21$ & $1.81 \pm 0.00$ & $1.66 \pm 0.21$ \\
\hline Camellia sinensis (fermented) & Chloroform F. & $1.20 \pm 0.43^{*}$ & $1.35 \pm 0.64^{*}$ & $1.35 \pm 0.64^{*}$ \\
\hline Camellia sinensis (fermented) & Methanol F. & $1.50 \pm 0.00$ & $1.81 \pm 0.00$ & $1.81 \pm 0.00$ \\
\hline Amantadine hydrochloride & - & $0.30 \pm 0.00^{* *}$ & $2.11 \pm 0.00$ & $2.11 \pm 0.00$ \\
\hline Oseltamivir carboxylate & - & $0.00 \pm 0.00^{* *}$ & $0.00 \pm 0.00^{* *}$ & $0.00 \pm 0.00^{* *}$ \\
\hline Influenza virus & - & $2.41 \pm 0.00$ & $2.41 \pm 0.00$ & $2.41 \pm 0.00$ \\
\hline
\end{tabular}

Data presented as mean \pm SD are averages of two independent $\mathrm{HA}$ titration which were tested in duplicates. ${ }^{*},{ }^{* *}$ : Significantly and highly significantly different from values obtained for compound-treated samples compared to untreated sample $(P \leq 0.05 \& P \leq 0.01)$ analyzed by SPSS, Tukey post-hoc test

which is indicative of HA physical interaction in all dilutions (Table 7).

\section{Preliminary phytochemical analysis results}

The existence of secondary metabolites was investigated by different preliminary analyses for the crude extracts, and chloroform and methanol fractions of five potent anti-influenza virus plants including G. glabra, M. communis, M. officinalis, S. alba and C. sinensis (fermented). Phytochemical analysis data (Table 8) confirmed the presence of alkaloids, cardiac glycosides, tannins, flavonoids, triterpenoids and steroids in the active crude extracts of G. glabra, M. officinalis and S. alba. The effective chloroform fractions of $M$. communis and C. sinensis (fermented) were rich in alkaloids, cardiac glycosides, triterpenoids and steroids. Methanol fractions of $M$. communis and $M$. officinalis with potential antiviral activities against influenza virus contained high amounts of flavonoids, tannins, triterpenoids and steroids.

\section{Discussion}

Medicinal plants have progressively been noticed as suitable alternatives to the synthetic antiviral agents [22-25]. In the current research, based on the antiviral properties of medicinal plants against IAV and other viruses, and their traditional and folklore usage in Iran, the antiviral efficacy of the crude extracts, and chloroform and methanol fractions of some Iranian native medicinal plants including G. glabra, M. communis, M. officinalis, $H$. perforatum, T. platyphyllos, S. alba, and C. sinensis (fermented and non-fermented) were evaluated against IAV with more details.

Previous investigation revealed that glycyrrhizin derived from the rhizomes of G. glabra has protective effects against IAV by induction of interferon [26]. In 
Table 4 Log HA decrement obtained from HA assay

\begin{tabular}{|c|c|c|c|c|}
\hline \multirow[t]{2}{*}{ Plant } & \multirow[t]{2}{*}{ Extracts or Fractions } & \multicolumn{3}{|c|}{$\begin{array}{l}\text { Log HA decrement } \\
\text { (mean } \pm \text { SD) }\end{array}$} \\
\hline & & Co-pen & Pre-pen & Post-pen \\
\hline Glycyrrhiza glabra & Crude E. & $5.00 \pm 4.24^{\mathrm{abc}}$ & $4.00 \pm 1.41^{b c}$ & $3.00 \pm 0.00^{\mathrm{abc}}$ \\
\hline Glycyrrhiza glabra & Chloroform F. & $1.00 \pm 0.00^{\mathrm{a}}$ & $1.00 \pm 0.00^{\mathrm{a}}$ & $2.00 \pm 1.41^{\mathrm{ab}}$ \\
\hline Glycyrrhiza glabra & Methanol F. & $5.50 \pm 3.54^{\mathrm{abc}}$ & $1.50 \pm 0.71^{\mathrm{a}}$ & $1.00 \pm 0.00^{\mathrm{a}}$ \\
\hline Myrtus communis & Crude E. & $2.50 \pm 0.71^{\mathrm{ab}}$ & $2.50 \pm 0.71^{\mathrm{ab}}$ & $2.50 \pm 0.71^{\mathrm{ab}}$ \\
\hline Myrtus communis & Chloroform F. & $5.50 \pm 3.54^{\mathrm{abc}}$ & $4.00 \pm 1.41^{b c}$ & $5.50 \pm 3.54^{c}$ \\
\hline Myrtus communis & Methanol F. & $3.00 \pm 1.41^{\mathrm{abc}}$ & $5.50 \pm 0.71^{c}$ & $4.00 \pm 0.00^{b c}$ \\
\hline Melissa officinalis & Crude E. & $3.50 \pm 0.71^{\mathrm{abc}}$ & $1.50 \pm 0.71^{\mathrm{a}}$ & $1.00 \pm 0.00^{\mathrm{a}}$ \\
\hline Melissa officinalis & Chloroform F. & $2.50 \pm 0.71^{\mathrm{ab}}$ & $2.50 \pm 0.71^{\mathrm{ab}}$ & $2.50 \pm 0.71^{\mathrm{ab}}$ \\
\hline Melissa officinalis & Methanol F. & $6.00 \pm 2.83^{\mathrm{abc}}$ & $1.00 \pm 0.00^{\mathrm{a}}$ & $1.50 \pm 0.71^{\mathrm{ab}}$ \\
\hline Hypericum perforatum & Crude E. & $3.00 \pm 1.41^{a b c}$ & $2.00 \pm 0.00^{\mathrm{a}}$ & $1.00 \pm 0.00^{\mathrm{a}}$ \\
\hline Hypericum perforatum & Chloroform F. & $2.50 \pm 0.71^{\mathrm{ab}}$ & $2.00 \pm 0.00^{\mathrm{a}}$ & $2.50 \pm 0.71^{\mathrm{ab}}$ \\
\hline Hypericum perforatum & Methanol F. & $3.00 \pm 1.41^{\mathrm{abc}}$ & $2.50 \pm 0.71^{\mathrm{ab}}$ & $3.00 \pm 1.41^{\mathrm{abc}}$ \\
\hline Tilia platyphyllos & Crude E. & $2.00 \pm 0.00^{\mathrm{ab}}$ & $2.00 \pm 0.00^{a b c}$ & $3.00 \pm 0.00^{\mathrm{abc}}$ \\
\hline Tilia platyphyllos & Chloroform F. & $3.00 \pm 1.41^{b}$ & $2.50 \pm 0.71^{\mathrm{abc}}$ & $3.00 \pm 0.00^{\mathrm{abc}}$ \\
\hline Tilia platyphyllos & Methanol F. & $4.00 \pm 0.00^{b}$ & $4.00 \pm 0.00^{c}$ & $2.00 \pm 0.00^{\mathrm{abc}}$ \\
\hline Salix alba & Crude E. & $4.00 \pm 1.41^{b}$ & $3.50 \pm 0.71^{b c}$ & $4.00 \pm 1.41^{c}$ \\
\hline Salix alba & Chloroform F. & $3.50 \pm 0.71^{b}$ & $2.00 \pm 1.41^{\mathrm{abc}}$ & $3.50 \pm 0.71^{b c}$ \\
\hline Salix alba & Methanol F. & $3.00 \pm 1.41^{b}$ & $2.50 \pm 2.12^{a b c}$ & $2.50 \pm 2.12^{\mathrm{abc}}$ \\
\hline Camellia sinensis & Crude E. & $0.50 \pm 0.71^{\mathrm{a}}$ & $1.50 \pm 0.71^{\mathrm{ab}}$ & $1.50 \pm 0.71^{\mathrm{abc}}$ \\
\hline Camellia sinensis & Chloroform F. & $2.00 \pm 0.00^{\mathrm{ab}}$ & $2.50 \pm 0.71^{\mathrm{ab}}$ & $1.00 \pm 1.41^{\mathrm{ab}}$ \\
\hline Camellia sinensis & Methanol F. & $0.50 \pm 0.71^{\mathrm{a}}$ & $0.50 \pm 0.71^{\mathrm{a}}$ & $3.00 \pm 0.00^{\mathrm{abc}}$ \\
\hline Camellia sinensis (fermented) & Crude E. & $2.50 \pm 0.71^{a b}$ & $2.00 \pm 0.00^{\mathrm{abc}}$ & $2.50 \pm 0.71^{\mathrm{abc}}$ \\
\hline Camellia sinensis (fermented) & Chloroform F. & $4.00 \pm 1.41^{b}$ & $3.50 \pm 2.121^{b c}$ & $3.50 \pm 2.12^{b c}$ \\
\hline Camellia sinensis (fermented) & Methanol F. & $3.00 \pm 0.00^{b}$ & $2.00 \pm 0.00^{\mathrm{abc}}$ & $2.00 \pm 0.00^{\mathrm{abc}}$ \\
\hline Amantadine hydrochloride & - & $7.00 \pm 0.00^{b c}$ & $1.00 \pm 0.00^{\mathrm{a}}$ & $1.00 \pm 0.00^{\mathrm{a}}$ \\
\hline Oseltamivir carboxylate & - & $8.00 \pm 0.00^{c}$ & $8.00 \pm 0.00^{d}$ & $8.00 \pm 0.00^{d}$ \\
\hline
\end{tabular}

Data presented as mean $\pm S D$ are averages of two independent HA titration which were tested in duplicates. Different letters show significant differences in each column (Duncan Grouping)

addition, other compounds of licorice showed significant inhibition on influenza A neuraminidase in a computerbased approach [27]. The effects of polyherbal formula containing licorice were confirmed for the prevention and treatment of influenza-like syndrome, clinically [28, 29].

M. officinalis essential oil could inhibit avian influenza virus (H9N2) through various replication cycle steps especially direct interaction with the virus particles [30]. Also, its extract demonstrated a significant anti-influenza effect against H1N1 strain of influenza virus [31].

The extract of $H$. perforatum showed anti-IAV effect both in vitro and in vivo. The $\mathrm{EC}_{50}$ of the extract was $40 \mu \mathrm{g} / \mathrm{ml}$ against IAV while its $\mathrm{CC}_{50}$ in MDCK cell line was $1.5 \mathrm{mg} / \mathrm{ml}$ [32]. In an experiment, it was observed that $H$. perforatum extract had significant efficacy for the treatment of mice infected with IAV [32]. In another study an opposite response occurred. The consumption of oral $H$. perforatum extract in the mice infected with influenza A virus, enhanced transcription of the suppressor of cytokine signaling 3 (SOCS3) and led to the impaired immune defense and higher mortality [33].

The anti-influenza activity of green tea (Camellia sinensis) against $\mathrm{H} 1 \mathrm{~N} 1$ virus was equivalent to green tea by-products $\left(\mathrm{EC}_{50}\right.$ equal to 6.72 and $6.36 \mu \mathrm{g} / \mathrm{ml}$, respectively). Also, hexane-soluble and ethyl acetate-soluble fractions of green tea by-products possessed strong anti-influenza activity in chickens [34]. The other studies demonstrated that dimeric polyphenol molecules in green tea display more potent antiviral effects against both influenza $\mathrm{A}$ and $\mathrm{B}$ viruses than monomers. In addition, the existance of $\mathrm{C}-4^{\prime}$ hydroxyl group in the $\mathrm{B}$ ring of planar flavonols is necessary for the anti-influenza $B$ virus activity $[35,36]$. It was confirmed clinically that formulations containing C. sinensis or green tea metabolites including catechines and theanine could prevent influenza infection $[37,38]$. 
Table 5 Cell viabilities from MTT assay in combined treatments with virus as compared to virus control group

\begin{tabular}{|c|c|c|c|c|}
\hline \multirow[t]{2}{*}{ Plant } & \multirow[t]{2}{*}{ Extracts or Fractions } & \multicolumn{3}{|l|}{$\begin{array}{l}\text { Cell viability } \\
\text { (mean } \pm \text { SD) }\end{array}$} \\
\hline & & Co-pen & Pre-pen & Post-pen \\
\hline Glycyrrhiza glabra & Crude E. & $0.42 \pm 0.04^{*}$ & $1.05 \pm 0.10^{* *}$ & $0.41 \pm 0.11^{* *}$ \\
\hline Glycyrrhiza glabra & Chloroform F. & $0.21 \pm 0.03$ & $0.21 \pm 0.03$ & $0.43 \pm 0.04^{* *}$ \\
\hline Glycyrrhiza glabra & Methanol F. & $0.86 \pm 0.29^{* *}$ & $0.71 \pm 0.10^{* *}$ & $0.36 \pm 0.11^{* *}$ \\
\hline Myrtus communis & Crude E. & $0.49 \pm 0.01^{* *}$ & $0.54 \pm 0.05^{* *}$ & $0.8 \pm 0.05^{* *}$ \\
\hline Myrtus communis & Chloroform F. & $0.77 \pm 0.11^{* *}$ & $0.49 \pm 0.04^{* *}$ & $0.42 \pm 0.11^{* *}$ \\
\hline Myrtus communis & Methanol F. & $0.20 \pm 0.01$ & $0.56 \pm 0.10^{* *}$ & $0.37 \pm 0.01^{* *}$ \\
\hline Melissa officinalis & Crude E. & $0.50 \pm 0.04^{* *}$ & $0.212 \pm 0.02$ & $0.29 \pm 0.10$ \\
\hline Melissa officinalis & Chloroform F. & $0.21 \pm 0.03$ & $1.01 \pm 0.31^{* *}$ & $0.32 \pm 0.04$ \\
\hline Melissa officinalis & Methanol F. & $0.81 \pm 0.05^{* *}$ & $0.45 \pm 0.06^{*}$ & $0.84 \pm 0.09^{* *}$ \\
\hline Hypericum perforatum & Crude E. & $0.51 \pm 0.03^{* *}$ & $0.35 \pm 0.01$ & $0.40 \pm 0.04^{* *}$ \\
\hline Hypericum perforatum & Chloroform F. & $0.62 \pm 0.12^{* *}$ & $0.62 \pm 0.11^{* *}$ & $0.91 \pm 0.04^{* *}$ \\
\hline Hypericum perforatum & Methanol F. & $0.21 \pm 0.03$ & $0.66 \pm 0.08^{* *}$ & $0.79 \pm 0.06^{* *}$ \\
\hline Tilia platyphyllos & Crude E. & $0.25 \pm 0.02$ & $0.21 \pm 0.01$ & $0.52 \pm 0.16^{* *}$ \\
\hline Tilia platyphyllos & Chloroform F. & $0.72 \pm 0.04^{* *}$ & $0.34 \pm 0.07$ & $0.68 \pm 0.07^{* *}$ \\
\hline Tilia platyphyllos & Methanol F. & $0.81 \pm 0.05^{* *}$ & $0.50 \pm 0.02^{* *}$ & $0.47 \pm 0.12^{* *}$ \\
\hline Salix alba & Crude E. & $0.80 \pm 0.07^{* *}$ & $0.73 \pm 0.03^{* *}$ & $0.533 \pm 0.08^{* *}$ \\
\hline Salix alba & Chloroform F. & $0.87 \pm 0.11^{* *}$ & $0.63 \pm 0.05^{* *}$ & $0.546 \pm 0.01^{* *}$ \\
\hline Salix alba & Methanol F. & $0.66 \pm 0.07^{* *}$ & $0.50 \pm 0.03$ & $0.420 \pm 0.07$ \\
\hline Camellia sinensis & Crude E. & $0.29 \pm 0.01$ & $0.39 \pm 0.09$ & $0.582 \pm 0.075^{* *}$ \\
\hline Camellia sinensis & Chloroform F. & $0.74 \pm 0.13^{* *}$ & $0.81 \pm 0.16^{* *}$ & $0.385 \pm 0.076$ \\
\hline Camellia sinensis & Methanol F. & $0.29 \pm 0.03$ & $0.26 \pm 0.01$ & $0.698 \pm 0.11^{* *}$ \\
\hline Camellia sinensis (fermented) & Crude E. & $0.87 \pm 0.11^{* *}$ & $0.74 \pm 0.13^{* *}$ & $0.581 \pm 0.03^{* *}$ \\
\hline Camellia sinensis (fermented) & Chloroform F. & $0.81 \pm 0.12^{* *}$ & $0.79 \pm 0.26^{* *}$ & $0.468 \pm 0.08^{* *}$ \\
\hline Camellia sinensis (fermented) & Methanol F. & $0.80 \pm 0.05^{* *}$ & $0.59 \pm 0.02^{* *}$ & $0.555 \pm 0.04^{* *}$ \\
\hline Amantadine hydrochloride & - & $1.01 \pm 0.03^{* *}$ & $0.59 \pm 0.05^{* *}$ & $0.61 \pm 0.06^{* *}$ \\
\hline Oseltamivir carboxylate & - & $1.11 \pm 0.01^{* *}$ & $1.06 \pm 0.01^{* *}$ & $1.04 \pm 0.03^{* *}$ \\
\hline Influenza virus & & $0.15 \pm 0.05$ & $0.15 \pm 0.05$ & $0.15 \pm 0.02$ \\
\hline
\end{tabular}

Data presented as mean $\pm S D$ are averages of two independent MTT assays which were tested in duplicates. **: highly significantly different from values obtained for drugs-treated samples compared to untreated sample $(P \leq 0.01)$ analyzed by SPSS, Tukey post-hoc test

According to the results, in terms of the selectivity index (SI), the extracts and fractions of all tested herbs were considered safe for the antiviral treatments except chloroform fraction of G. glabra. Its SI value was the same as conventional drugs. In addition, $C$. sinensis, $S$. alba, $H$. perforatum and M. officinalis were categorized as the safest plants in cellular studies.

In HI test, the extracts of T. platyphyllos, S. alba and C. sinensis showed RBC precipitation in all tested dilutions which indicates the strong physical interaction of these compounds with the HA surface glycoprotein of the virus. However, amongst the others, methanol fraction of M. officinalis showed the weakest interaction (3rd dilution), and on the opposite side M. communis and H. perforatum showed stronger interaction (5th dilution). The results of this study confirmed dose-dependent response for most of the extracts and fractions. In a previous research, the formation of complexes between tannins and proteins was confirmed [39]. Also it was demonstrated that antiviral inhibitory effects of hydrolyzable tannins were related to the intractions blocking between viral glycoproteins and cell surface glycosaminoglycans (GAGs) [40]. It is interesting that in our experiment, $M$. officinalis, unlike the others, did not contain any tannin which justificated the weakest intraction.

With a thorough scrutiny on the Tables 3, 4, 5 and 6 and comparing their results with the SI values in Table 2 and the fact that SI values higher than 3 are safe compounds, it was concluded that except one almost all tested samples were safe but not effective against IAV titer.

Moreover, General Linear Model (GLM) analysis which estimated marginal means of all the respective values for different exposure ways (combined treatments) confirmed all the outcomes. The data are shown in the Supplementary Figs. 1, 2, 3 and 4. Therefore, most of the above mentioned plant species might be promising 
Table 6 Cellular percentage of protection in combined treatments with virus as compared to control groups

\begin{tabular}{|c|c|c|c|c|}
\hline \multirow[t]{2}{*}{ Plant } & \multirow[t]{2}{*}{ Extracts or Fractions } & \multicolumn{3}{|c|}{$\begin{array}{l}\text { Percentage of protection } \\
\text { (mean } \pm \text { SD) }\end{array}$} \\
\hline & & Co-pen & Pre-pen & Post-pen \\
\hline Glycyrrhiza glabra & Crude E. & $24.75 \pm 3.88$ & $88.99 \pm 10.53$ & $23.02 \pm 10.91$ \\
\hline Glycyrrhiza glabra & Chloroform F. & $3.24 \pm 3.20$ & $3.33 \pm 3.38$ & $25.78 \pm 4.40$ \\
\hline Glycyrrhiza glabra & Methanol F. & $69.50 \pm 29.42$ & $53.93 \pm 10.02$ & $18.77 \pm 10.75$ \\
\hline Myrtus communis & Crude E. & $31.52 \pm 0.34$ & $36.67 \pm 4.95$ & $71.06 \pm 4.94$ \\
\hline Myrtus communis & Chloroform F. & $60.04 \pm 11.41$ & $31.61 \pm 4.32$ & $24.42 \pm 10.55$ \\
\hline Myrtus communis & Methanol F. & $2.02 \pm 0.69$ & $38.70 \pm 9.90$ & $19.43 \pm 0.21$ \\
\hline Melissa officinalis & Crude E. & $33.01 \pm 4.32$ & $3.20 \pm 1.73$ & $11.32 \pm 10.36$ \\
\hline Melissa officinalis & Chloroform F. & $2.89 \pm 2.80$ & $84.29 \pm 31.20$ & $14.39 \pm 3.67$ \\
\hline Melissa officinalis & Methanol F. & $64.57 \pm 5.12$ & $27.42 \pm 6.20$ & $67.66 \pm 8.87$ \\
\hline Hypericum perforatum & Crude E. & $33.78 \pm 3.31$ & $17.81 \pm 0.79$ & $21.99 \pm 4.20$ \\
\hline Hypericum perforatum & Chloroform F. & $45.21 \pm 12.37$ & $44.95 \pm 11.18$ & $74.80 \pm 3.92$ \\
\hline Hypericum perforatum & Methanol F. & $3.04 \pm 3.27$ & $48.61 \pm 8.44$ & $62.56 \pm 6.23$ \\
\hline Tilia platyphyllos & Crude E. & $10.44 \pm 3.85$ & $3.89 \pm 1.57$ & $48.37 \pm 22.95$ \\
\hline Tilia platyphyllos & Chloroform F. & $77.17 \pm 5.54$ & $23.35 \pm 10.03$ & $71.12 \pm 9.35$ \\
\hline Tilia platyphyllos & Methanol F. & $90.78 \pm 7.56$ & $45.77 \pm 3.50$ & $41.79 \pm 17.84$ \\
\hline Salix alba & Crude E. & $88.27 \pm 9.67$ & $78.03 \pm 4.47$ & $50.48 \pm 11.32$ \\
\hline Salix alba & Chloroform F. & $98.71 \pm 17.46$ & $64.78 \pm 6.56$ & $52.35 \pm 14.25$ \\
\hline Salix alba & Methanol F. & $68.60 \pm 10.69$ & $38.46 \pm 3.80$ & $34.24 \pm 9.92$ \\
\hline Camellia sinensis & Crude E. & $16.03 \pm 0.86$ & $30.29 \pm 13.24$ & $57.43 \pm 10.70$ \\
\hline Camellia sinensis & Chloroform F. & $80.67 \pm 18.75$ & $95.54 \pm 15.29$ & $29.40 \pm 10.98$ \\
\hline Camellia sinensis & Methanol F. & $16.24 \pm 0.39$ & $11.36 \pm 0.85$ & $74.05 \pm 15.81$ \\
\hline Camellia sinensis (fermented) & Crude E. & $99.40 \pm 16.05$ & $80.63 \pm 19.28$ & $57.27 \pm 4.49$ \\
\hline Camellia sinensis (fermented) & Chloroform F. & $90.46 \pm 16.83$ & $57.23 \pm 36.94$ & $41.18 \pm 11.35$ \\
\hline Camellia sinensis (fermented) & Methanol F. & $90.58 \pm 9.05$ & $58.85 \pm 2.59$ & $53.53 \pm 5.45$ \\
\hline Amantadine hydrochloride & - & $83.54 \pm 3.16$ & $42.25 \pm 4.76$ & $43.66 \pm 5.76$ \\
\hline Oseltamivir carboxylate & - & $94.78 \pm 0.40$ & $89.63 \pm 0.81$ & $87.33 \pm 3.33$ \\
\hline
\end{tabular}

alternatives to decrease flu unfavorable effects by affecting the viral and cellular receptors. The data were considerable because the conventional antiviral drugs; amantadine and oseltamivir showed promising effects against virus infection, however, growing drug resistance has caused a significant challenge [41, 42].

The phytochemical analysis of the potent anti-IAV extracts/fractions demonstrated that they were rich in flavonoids, tannins, triterpenoids and steroids. Natural products have different mechanisms against viral infections from interfering with entry, transcription, replication and translation of the virus, nuclear export of the virus, viral assembly, packing and budding to enhance the host responses [43].

There were many reports that showed flavonids act as anti-IAV compounds with various mechanisms [44, 45]. Flavonoids are natural phenolic compounds of plants with potent antioxidant and antiviral properties. They can help viral-infected cells to fix their biochemical imbalance resulting from oxidative stress [46]. Also they have shown potential inhibition on the neuraminidase active site of influenza virus. The potency of NA blocking reduced from aurones to flavon(ol)es, isoflavones, flavanon(ol)es and flavan(ol)es, respectively. The structure activity relationship (SAR) studies of flavonoids against influenza virus demonstrated that the presence of 7-OH, $4^{\prime}-\mathrm{OH}, \mathrm{C}_{2}=\mathrm{C}_{3}$ and $\mathrm{C}_{4}=\mathrm{O}$ functionalities were necessary, but the existance of a sugar group reduced the effects $[47,48]$.

Triterpenoids and steroids are natural components elucidated from plants and other organisms which have various biological activities including antiviral activities. Mechanistic studies revealed triterpenoids bind tightly to the viral hemagglutinin (HA) and disrupt the attachment of viruses to the cell receptors [49-51].

Influenza virus has two envelope glycoproteins named hemagglutinin (HA) and neuraminidase (NA). The binding of hemagglutinin to sialic acid residues of the host cells is a key step for initiating the IAV infection. The role of NA is facilitating the movement of virus from infection 
Table 7 Dose-dependent response

\begin{tabular}{|c|c|c|}
\hline Plant & Extracts or Fractions & $\begin{array}{l}\text { Dilution } \\
\text { to give } \\
\mathrm{HI}+\end{array}$ \\
\hline Glycyrrhiza glabra & Crude E. & 4th \\
\hline Glycyrrhiza glabra & Chloroform F. & 4 th \\
\hline Glycyrrhiza glabra & Methanol F. & 4 th \\
\hline Myrtus communis & Crude E. & 5 th \\
\hline Myrtus communis & Chloroform F. & 4th \\
\hline Myrtus communis & Methanol F. & 4 th \\
\hline Melissa officinalis & Crude E. & - \\
\hline Melissa officinalis & Chloroform F. & - \\
\hline Melissa officinalis & Methanol F. & $3 \mathrm{rd}$ \\
\hline Hypericum perforatum & Crude E. & $3 \mathrm{rd}$ \\
\hline Hypericum perforatum & Chloroform F. & 5 th \\
\hline Hypericum perforatum & Methanol F. & $3 r d$ \\
\hline Tilia platyphyllos & Crude E. & - \\
\hline Tilia platyphyllos & Chloroform F. & - \\
\hline Tilia platyphyllos & Methanol F. & - \\
\hline Salix alba & Crude E. & - \\
\hline Salix alba & Chloroform F. & - \\
\hline Salix alba & Methanol F. & - \\
\hline Camellia sinensis & Crude E. & - \\
\hline Camellia sinensis & Chloroform F. & - \\
\hline Camellia sinensis & Methanol F. & - \\
\hline Camellia sinensis (fermented) & Crude E. & - \\
\hline Camellia sinensis (fermented) & Chloroform F. & - \\
\hline Camellia sinensis (fermented) & Methanol F. & - \\
\hline Amantadine hydrochloride & - & $3 \mathrm{rd}$ \\
\hline Oseltamivir carboxylate & - & $3 r d$ \\
\hline
\end{tabular}

sites to the respiratory tract $[52,53]$. The presence of flavonoids, tannins, steroids and triterpenoids together in the potent anti-influenza extracts and fractions of this study, covers the inhibitory effects on both the HA and NA may be a reason for such significant effects. It is good to note that the virus strain used in this study, $\mathrm{A} / \mathrm{PR} / 8 / 34$, is not pathogenic to humans and may not be the best model for these types of studies. However, this strain is generally used because it provides acceptable oucomes comparable to the pathogenic strains and is also safe for the users.

\section{Conclusions}

The emergence of new strains of the influenza A virus makes us think about innovative strategies for the development of new drugs with improved antiviral effects, higher safety and better tolerability. Research focusing on traditional herbs as complementary therapies or preventive medicine is becoming more attractive. Medicinal plants have been used for various ailments, particularly infectious diseases. The current study indicated that treatment of IAV with the selected extracts and fractions reduced the hemagglutination activity of the virus, which may result from the physical interaction of the samples with virus hemagglutinin. Based on this scientific confirmation, the selected plants may have the capacity to ease the symptoms and burden of flu. The next focus of this study will be the purification of pure compounds responsible for the bioactivity against IAV infection as well as their mode of action. Recommendations are proposed for strategizing the future role and place of medicinal plants in prevention and treatment of influenza and other infectious

Table 8 Preliminary phytochemical analysis of crude extracts and different fractions of five potential plants with anti-influenza A activity

\begin{tabular}{|c|c|c|c|c|c|c|c|c|c|c|c|c|c|c|c|c|}
\hline \multirow[t]{3}{*}{ Phytochemical tests } & & \multicolumn{3}{|c|}{$\begin{array}{l}\text { Glycyrrhiza } \\
\text { glabra }\end{array}$} & \multicolumn{3}{|c|}{$\begin{array}{l}\text { Myrtus } \\
\text { commonis }\end{array}$} & \multicolumn{3}{|c|}{$\begin{array}{l}\text { Melissa } \\
\text { officinalis }\end{array}$} & \multicolumn{3}{|c|}{$\begin{array}{l}\text { Salix } \\
\text { alba }\end{array}$} & \multicolumn{3}{|c|}{$\begin{array}{l}\text { Camellia } \\
\text { sinensis } \\
\text { (fermented) }\end{array}$} \\
\hline & & $C$ & $C$ & $M$ & $C$ & $C$ & $M$ & $C$ & $C$ & $M$ & $C$ & $C$ & $M$ & $C$ & $C$ & $M$ \\
\hline & & $\mathrm{E}$ & $F$ & $F$ & $\mathbf{E}$ & $F$ & $\mathbf{F}$ & $E$ & $F$ & $F$ & 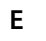 & $F$ & $\mathbf{F}$ & $\mathrm{E}$ & $F$ & $F$ \\
\hline \multirow[t]{2}{*}{ Alkaloids } & Dragendorff's Test & - & - & - & + & + & - & + & + & - & + & - & + & + & + & - \\
\hline & Hager's Test & - & - & - & + & - & + & + & + & - & + & + & - & + & + & - \\
\hline Cardiac Glycosides & Keller-killiani & + & - & + & + & + & - & + & + & - & + & - & + & + & + & - \\
\hline \multirow[t]{2}{*}{ Tannins } & Ferric Chloride Test & + & - & + & + & - & + & - & - & - & + & - & + & + & - & + \\
\hline & Gelatin test & + & + & + & + & - & + & - & - & - & + & - & + & + & - & + \\
\hline Flavonoids & Shinoda Test & + & - & + & + & - & + & + & - & + & + & - & + & + & - & + \\
\hline \multirow[t]{2}{*}{ Steroids and Triterpenoids } & Salkowski test & + & + & - & + & + & + & + & - & + & + & + & - & + & + & + \\
\hline & Libermann-Buchard test & - & - & - & - & + & - & + & + & + & - & + & - & - & + & - \\
\hline Saponin & Foam test & - & - & - & - & - & - & - & - & - & - & - & - & - & - & - \\
\hline
\end{tabular}


diseases in national, regional and international health policies and programs.

\begin{abstract}
Abbreviations
ANOVA: Analysis of variance; $C_{C I D}$ : Cell culture infectious dose $50 ; C_{50}: 50 \%$ cytotoxic concentration; CRBCs: Chicken red blood cells; DMEM: Dulbecco's Modified Eagle's Medium; $\mathrm{EC}_{50}$ : 50\% effective concentration; FBS: Fetal Bovine Serum; GLM: General Linear Model; HA: Hemagglutination Assay; HI: Hemagglutination Inhibition Assay; IAV: Influenza A Virus; MDCK: Madin Darby Canine Kidney; MTT: [3-(4,5-dimethyl-2-thiazolyl)-2,5-diphenyl-2H-tetrazolium bromide]; OD: Optical Density; SI: Selectivity Index; Trypsin-TPCK: Tosylamide Phenylethyl Chloromethyl Keton-treated Trypsin.
\end{abstract}

\section{Supplementary Information}

The online version contains supplementary material available at https://doi. org/10.1186/s12906-021-03423-X.

Additional file 1: Supplementary Fig. 1. Estimated marginal means of Log HA titer. This graph shows the Log HA titer levels analyzed by GLM. Glycyrrhiza glabra crude extract (1), Glycyrrhiza glabra methanol fraction (2), Glycyrrhiza glabra chloroform fraction (3), Myrtus communis crude extract (4), Myrtus communis methanol fraction (5), Myrtus communis chloroform fraction (6), Melissa officinalis crude extract (7), Melissa officinalis methanol fraction (8), Melissa officinalis chloroform fraction (9), Hypericum perforatum crude extract (10), Hypericum perforatum methanol fraction (11), Hypericum perforatum chloroform fraction (12), Tiliatilia platyphyllos crude extract (13), Tilia platyphyllos methanol fraction (14), Tilia platyphyllos chloroform fraction (15), Salix alba crude extract (16), Salix alba methanol fraction (17), Salix alba chloroform fraction (18), Camellia sinensis crude extract (19), Camellia sinensis methanol fraction (20), Camellia sinensis chloroform fraction (21), Camellia sinensis fermented crude extract (22), Camellia sinensis fermented methanol fraction (23), Camellia sinensis fermented chloroform fraction (24), Amantadine hydrochloride (25), Oseltamivir carboxylate (26), IAV (27), 1 (blue): Co-penetration, 2(green): Pre-penetration, 3 (brown): Post-penetration.

Additional file 2: Supplementary Fig. 2. Estimated marginal means of Log HA decrement. This graph shows the decrement levels in Log HA titers analyzed by GLM.

Additional file 3: Supplementary Fig. 3. Estimated marginal means of cell viability. This graph shows the ODs of the cell viability test analyzed by GLM.

Additional file 4: Supplementary Fig. 4. Estimated marginal means of the percentage of protection. This graph shows the protection of the extracts on the cell viability analyzed by GLM.

\section{Acknowledgements}

Our sincere gratitude goes to the staff of Influenza and Respiratory Viruses Department, Pasteur Institute of IRAN. We also thank Laboratory of Vaccine and Immunotherapeutics, Institute of Bioscience, Universiti Putra Malaysia; Prof Dr. Aini Ideris and Prof Dr. Abdul Rahman Omar who kindly provided amantadine hydrochloride and oseltamivir carboxylate.

\section{Authors' contributions}

PM, ZT, SG co-defined the research theme. PM designed the methods and experiments. ZT, SG provided the facilities of the Phytochemical Laboratory. FF provided the facilities for antiviral work. SG, YM and HE prepared plants, extracts and fractions. PM, HS, ZM carried out the laboratory experiments for IAV. PM analyzed the data, drafted the manuscript and worked on the associated data collection and their interpretation. PM, ZT, SG revised the manuscript critically for editorial and important intellectual content. All authors have seen and approved the manuscript.

\section{Funding}

Not Applicable.

\section{Availability of data and materials}

All data and materials are contained and described within the manuscript. The datasets used and/or analyzed during the current study are available from the corresponding author on reasonable request.

\section{Declarations}

Ethics approval and consent to participate

This research achieved ethical codes from Pharmaceutical Sciences Research Center, Tehran University of Medical Sciences (IR.TUMS.PSRC.REC.1397.5023 \& IR.TUMS.TIPS.REC.1397.028).

\section{Consent for publication}

Not applicable.

\section{Competing interests}

The authors declare that they have no competing interests.

\section{Author details}

${ }^{1}$ Influenza and Respiratory Viruses Department, Pasteur Institute of IRAN, Tehran, Iran. ${ }^{2}$ Department of Pharmacognosy, Faculty of Pharmacy, Tehran University of Medical Sciences, Tehran, Iran. ${ }^{3}$ Medicinal Plants Research Center, Faculty of Pharmacy, Tehran University of Medical Sciences, Tehran, Iran.

Received: 10 October 2019 Accepted: 24 September 2021

Published online: 01 October 2021

\section{References}

1. Webster R, Bean WGO, Chambers T, Kawaoka Y. Evolution and ecology of influenza A viruses. Microbiol Rev. 1992;56:152-79.

2. Fedson DS. Confronting an influenza pandemic with inexpensive generic agents: can it be done? Lancet Infect Dis. 2008;8:571-6.

3. Cox NM. Pandemic influenza: overview of vaccines and antiviral drugs. Yale J Biol Med. 2005;78:321-8.

4. Scholtissek C, Quack G, Klenk HD, Webster RG. How to overcome resistance of influenza A viruses against adamantane derivatives. Antiviral Res. 1998:37:83-95.

5. Saladino R, Barontini M, Nencioni M, Sgarbanti R, Palamara AT. Current advances in anti-influenza therapy. Curr Med Chem. 2010;17:2101-40.

6. Ison MG. Antivirals and resistance: Influenza virus. Curr Opin Virol. 2011;1:563-73.

7. Pathumwadee I, Chittima L, Thanyada R, Arthorn L, Maturos M, Panita D, et al. How amantadine and rimantadine inhibit proton transport in the M2 protein channel. J Mol Graph Model. 2008;27:342-8.

8. Sarah CD, Hong M. Amantadine-induced conformational and dynamical changes of the influenza M2 transmembrane proton channel. P Natl Acad Sci USA. 2008;105:1483-8.

9. Arakawa T, Yamasaki H, Ikeda K, Ejima D, Naito T, Koyama AH. Antiviral and virucidal activities of natural product. Curr Med Chem. 2009;16:2485-97.

10. Khalafalla MM, Abdellatef E, Dafalla HM, Nassrallah AA, Aboul-Enein KM, Lightfoot DA, et al. Active principle from moringa oleifera Lam leaves effective against two leukemias and a hepatocarcinoma. Afr J Biotechnol. 2010;9:8467-71.

11. Goodarzi S, Nateghpour M, Asgharian P, Hadjiakhoondi A, Yassa N, Tavakoli S, et al. Antimalarial and cytotoxic activities of roots and fruits fractions of Astrodaucus persicus extract. Iran J Basic Med Sci. 2017;20:1318-23.

12. Aqil F, Ahmad I, Mehmood Z. Antioxidant and free radical scavenging properties of twelve traditionally used Indian medicinal plants. Turk J Biology. 2006;30:177-83.

13. Kang $Q$, Wang $Y$, Cui $Q$, Gong L, Yang Y, Jiang $H$, et al. Screening for antiinfluenza actives of orefractionated traditional Chinese medicines. Evid Based Complement Alternat Med. 2020;2020:4979850.

14. Shahzad Ml, Ashraf H, Aslam A, Parveen S, Kamran Z, Naz N, et al. REPORT- Some ethanobotanically important plants from Cholistan area for anti avian influenza virus (AIV) H9N2 screening. Pak J Pharm Sci. 2019;32:2751-6. 
15. Karber G. 50\% endpoint calculation. Arch Exp Pathol Pharmacol. 1931;162:480-3.

16. Mehrbod P, Abdalla MA, Njoya EM, Ahmed AS, Fotouhi F, Farahmand B, et al. South African medicinal plant extracts active against influenza $A$ virus. BMC CAM. 2018;18:1-10.

17. Chattopadhyay D, Sarkar MC, Chatterjee T, Sharma Dey R, Bag P, Chakraborti S, et al. Recent advancements for the evaluation of anti-viral activities of natural products. New Biotechnol. 2009;25:347-68.

18. Shigeta S, Shuichi M, Junko W, Shu S, Kauzo T, Yamase T. Synergistic antiinfluenza virus A (H1N1) activities of PM-523 (Polyoxomatalate) and Ribavirin in vitro and in vivo. Antimicrob Agents Ch. 1997;41:423-1427.

19. Hirst GK. The quantitative determination of influenza virus and antibodies by means of red cell agglutination. J Exp Med. 1942;75:49-64.

20. Rimjhim S, Kumari N, Jainendra K. Preliminary phytochemical screening of methanolic extract of clerodendron infortunatum. IOSR J Appl Chem. 2014;7:10-3.

21. Heshmati Afshar F, Delazar A, Asnaashari S, Vaez H, Zolali E, Asgharian P. Screening of anti-malarial activity of different extracts obtained from three species of Scrophularia growing in Iran. Iran J Pharm Res. 2018;17:668-76

22. Camargo F, Cortez DA, Ueda NT, Nakamura CV, Dias Filho BP. Antiviral activity and mode of action of a peptide isolated from Sorghum bicolor. Phytomedicine. 2008;15:202-8.

23. Choi HJ, Lim CH, Song JH, Baek SH, Kwon DH. Antiviral activity of raoulic acid from Raoulia australis against picornaviruses. Phytomedicine. 2009;16:35-9.

24. Rajasekaran D, Palombo EA, Chia Yeo T, Lim Siok Ley D, Lee Tu C, Malherbe $F$, et al. Identification of traditional medicinal plant extracts with novel anti-influenza activity. PLOS ONE. 2013:8:e79293.

25. Mehrbod P, Ideris A, Omar AR, Hair-Bejo M, Tan SW, Kheiri MT, et al. Attenuation of influenza virus infectivity with herbal-marine compound (HESA-A): an in vitro study in MDCK cells. Virol J. 2012;9:44.

26. Fiore $C$, Eisenhut $M$, Krausse R, Ragazzi E, Pellati D, Armanini D, et al. Antiviral effects of Glycyrrhiza species. Phytother Res. 2008;22:141-8.

27. Grienke U, Braun H, Seidel N, Kirchmair J, Richter M, Krumbholz A, et al. Computer-guided approach to access the anti-influenza activity of licorice constituents. J Nat Prod. 2014;77:563-70.

28. Clement $Y$, Leung PC. Effects of a polyherbal formula on influenza-like syndrome and immune responses. Focus Altern Complement Ther. 2013:18:207-8.

29. Wong LY, Leung PC, Pang SY, Cheng KF, Wong CK, Lam WK, et al. A herbal formula for prevention of influenza-like syndrome: A double-blind randomized clinical trial. Chin J Integr Med. 2013;19:253-9.

30. Pourghanbari G, Nili H, Moattari A, Mohammadi A, Iraji A. Antiviral activity of the oseltamivir and Melissa officinalis L. essential oil against avian influenza A virus (H9N2). Virusdisease. 2016:27:170-8.

31. Jalali P, Moattari A, Mohammadi A, Ghazanfari N, Pourghanbari G. Melissa officinalis efficacy against human influenza virus (New H1N1) in comparison with oseltamivir. Asian Pac JTrop Dis. 2016;6:714-7.

32. Pu XY, Liang JP, Wang XH, Xu T, Hua LY, Shang RF, et al. Anti-influenza A virus effect of Hypericum perforatum L. extract. Virologica Sinica. 2009;24:19.

33. Huang N, Singh N, Yoon K, Loiacono CM, Kohut ML, Birt DF. The immunoregulatory impact of orally-administered Hypericum perforatum extract on Balb/C mice inoculated with H1n1 influenza A virus. PLOS ONE. 2013;8:e76491.

34. Lee HJ, Lee YN, Youn HN, Lee DH, Kwak JH, Seong BL, et al. Anti-influenza virus activity of green tea by-products in vitro and efficacy against influenza virus infection in chickens. Poult Sci. 2012;91:66-73.

35. Yang ZF, Bai LP, Huang WB, Li X-Z, Zhao SS, Zhong NS, et al. Comparison of in vitro antiviral activity of tea polyphenols against influenza $A$ and $B$ viruses and structure-activity relationship analysis. Fitoterapia. 2014;93:47-53.
36. Zu M, Yang F, Zhou W, Liu A, Du G, Zheng L. In vitro anti-influenza virus and anti-inflammatory activities of theaflavin derivatives. Antiviral Res. 2012:94:217-24.

37. Matsumoto K, Yamada H, Takuma N, Niino H, Sagesaka YM. Effects of green tea catechins and theanine on preventing influenza infection among healthcare workers: A randomized controlled trial. BMC CAM. 2011;11:1-7.

38. Rowe CA, Nantz MP, Bukowski JF, Percival SS. Specific formulation of Camellia sinensis prevents cold and flu symptoms and enhances gamma,delta $T$ cell function: a randomized, double-blind, placebo-controlled study. J Am Coll Nutr. 2007;26:445-52.

39. Hagerman AE, Rice ME, Ritchard NT. Mechanisms of protein precipitation for two tannins, pentagalloyl glucose and epicatechin16 $(4 \rightarrow 8)$ catechin (procyanidin). J Agric Food Chem. 1998;46:2590-5.

40. Liang-Tzung L, Ting-Ying C, Song-Chow L, Chueh-Yao C, Ta-Chen L, GueyHorng W, et al. Broad-spectrum antiviral activity of chebulagic acid and punicalagin against viruses that use glycosaminoglycans for entry. BMC Microbiol. 2013;13:45

41. Hayden FG, de Jong MD. Emerging influenza antiviral resistance threats. J Infect Dis. 2011;203:6-10

42. Cheng PKC, Leung TWC, Ho ECM, Leung PCK, Ng AYY, Lai MYY, et al. Oseltamivir- and amantadine-resistant influenza viruses A (H1N1). Emerg Infect Diseases. 2009;15:966-8.

43. Tsang N, Zhao L, Tsang S, Zhang H. Antiviral activity and molecular targets of plant natural products against avian influenza virus. Curr Org Chem. 2017;21:1777-804.

44. Mohan S, Elhassan Taha MM, Makeen HA, Alhazmi HA, Al Bratty M, Sultana S, et al. Bioactive natural antivirals: An updated review of the available plants and isolated molecules. Molecules. 2020;25:E4878.

45. Zhang J, Wang Y. Bilobetin, a novel small molecule inhibitor targeting influenza virus polymerase acidic (PA) endonuclease was screened from plant extracts. Nat Prod Res. 2020;21:1-4.

46. Diniz LRL, Bezerra Filho CSM, Fielding BC, de Sousa DP. Natural antioxidants: A review of studies on human and animal coronavirus. Oxid Med Cell Longev. 2020;2020:3173281.

47. Sadati SM, Gheibi N, Ranjbar S, Hashemzadeh MS. Docking study of flavonoid derivatives as potent inhibitors of influenza H1N1 virus neuraminidase. Biomed Rep. 2019;10:33-8.

48. Liu AL, Wang HD, Lee SM, Wang YT, Du GH. Structure-activity relationship of flavonoids as influenza virus neuraminidase inhibitors and their in vitro anti-viral activities. Bioorg Med Chem. 2008;16:7141-7.

49. Yu M, Si L, Wang YT, Wu Y, Yu F, Jiao P, et al. Discovery of pentacyclic triterpenoids as potential entry inhibitors of influenza viruses. J Med Chem. 2014;57:10058-71.

50. Xiao S, Tian Z, Wang YT, Si L, Zhang L, Zhou D. Recent progress in the antiviral activity and mechanism study of pentacyclic triterpenoids and their derivatives. Med Res Rev. 2018:38:951-76.

51. Vil VA, Terent'ev AO, Savidov N, Gloriozova TA, Poroikov VV, Pounina TA, et al. Hydroperoxy steroids and triterpenoids derived from plant and fungi: Origin, structures and biological activities. J Steroid Biochem Mol Biol. 2019;190:76-87.

52. Ruikun D, Qinghua C, Lijun R. Competitive cooperation of hemagglutinin and neuraminidase during influenza A virus entry. Viruses. 2019:11:458.

53. Mercader AG, Pomilio AB. Study of flavonoids and biflavonoids as influenza H1N1 virus neuraminidase inhibitors. Eur J Med Chem. 2010;45:1724-30.

\section{Publisher's Note}

Springer Nature remains neutral with regard to jurisdictional claims in published maps and institutional affiliations. 